\title{
ABSTRACT $\omega$-LIMIT SETS, CHAIN RECURRENT SETS, AND BASIC SETS FOR FLOWS
}

\author{
JOHN E. FRANKE AND JAMES F. SELGRADE
}

ABstract. An abstract $\omega$-limit set for a flow is an invariant set which is conjugate to the $\omega$-limit set of a point. This paper shows that an abstract $\omega$ limit set is precisely a connected, chain recurrent set. In fact, an abstract ${ }^{-} \omega$ limit set which is a subset of a hyperbolic invariant set is the $\omega$-limit set of a nearby heteroclinic point. This leads to the result that a basic set is a hyperbolic, compact, invariant set which is chain recurrent, connected, and has local product structure.

1. Introduction. $R$. Bowen [1] defines a homeomorphism on a compact metric space to be an abstract $\omega$-limit set if it is conjugate to the $\omega$-limit set of some point. He shows that if $f$ is an Axiom $\mathbf{A}$ diffeomorphism and if $f$ restricted to $\Lambda$, a subset of the nonwandering set $\Omega$, is an abstract $\omega$-limit set then $\Lambda=\omega(x)$ for some $x \in \Omega$. In this paper we investigate related questions for flows.

Definition. A flow $f$ on $\Lambda$ is an abstract $\omega$-limit set if there is a flow $g$ on $X$ a compact metric space and an $x \in X$ so that $\left.g\right|_{\omega(x)}$ is topologically conjugate to $f$.

C. Conley [2] defines a weak form of recurrence, called chain recurrence, for a flow $f$ on a compact metric space $M$. The set of points with this recurrence property is called the chain recurrent set $R(f)$. If $\mathcal{R}(f)=M$ then $f$ is said to be chain recurrent.

Theorem A. A flow $f$ on $\Lambda$ is an abstract $\omega$-limit set if and only if $\Lambda$ is connected and $f$ is chain recurrent.

THeOREM B. Let $f$ be a smooth flow on $M$ and let $\Lambda$ be a hyperbolic closed invariant subset of $M$. If $\left.f\right|_{\Lambda}$ is an abstract $\omega$-limit set and if $\alpha>0$, then there is an $x \in W_{\alpha}^{u}(\Lambda) \cap W_{\alpha}^{s}(\Lambda)$ such that $\omega(x)=\alpha(x)=\Lambda$. $\left(W_{\alpha}^{s}(\Lambda)\right.$ and $W_{\alpha}^{u}(\Lambda)$ denote local stable and unstable manifolds of $\Lambda$.)

If $\Lambda$ has local product structure (i.e., $W_{\alpha}^{s}(\Lambda) \cap W_{\alpha}^{u}(\Lambda)=\Lambda$ ) in addition to the hypothesis in Theorem $\mathrm{B}$, then this $x$ is a point in $\Lambda$ and its orbit is dense in $\Lambda$. In the case that $f$ is an Axiom A flow, $\Omega$ has local product structure [5] so Theorem B gives the flow version of Bowen's result.

S. Smale [6] defines a basic set for a flow $f$ on $M$ to be a set $\Lambda$ such that

Received by the editors January 19, 1976.

AMS (MOS) subject classifications (1970). Primary 58F99; Secondary 58F15.

Key words and phrases. Flows, abstract $\omega$-limit set, chain recurrent, invariant set, hyperbolic, Axiom A, basic set. 
(a) $\Lambda$ is compact and invariant,

(b) $\Lambda$ is hyperbolic,

(c) periodic orbits are dense in $\Lambda$,

(d) $\Lambda$ has a transitive orbit,

(e) there is an open neighborhood $U$ of $\Lambda$ such that $\bigcap_{t \in R} f_{t}(U)=\Lambda, U$ is called a fundamental neighborhood.

We are able to weaken several of Smale's conditions and still obtain an equivalent definition of a basic set.

THEOREM C. $\Lambda$ is a basic set for a flow $f$ if and only if

( $\left.\mathrm{a}^{\prime}\right) \Lambda$ is compact and invariant,

(b') $\Lambda$ is hyperbolic,

$\left.\left(c^{\prime}\right) f\right|_{\Lambda}$ is chain recurrent,

(d') $\Lambda$ is connected,

(e') $\Lambda$ has local product structure.

Theorem B with local product structure gives (d); Proposition 2.3 in $\$ 2$ with local product structure gives (c); and (e) follows from

Proposition D. If $\Lambda$ is a hyperbolic closed invariant set with local product structure then it has a fundamental neighborhood.

2. Background and notation. Let $f$ be a flow on a compact metric space $(M, d)$. For subsets $\Lambda$ of $M$ and $J$ of $R$, define $\Lambda \cdot J=f(\Lambda \times J)$. Given $\varepsilon, T>0$, an infinite $(\varepsilon, T)$-chain is a pair of doubly infinite sequences

$$
\left\{\cdots, x_{-2}, x_{-1}, x_{0}, x_{1}, x_{2}, \ldots ; \ldots, t_{-2}, t_{-1}, t_{0}, t_{1}, t_{2}, \ldots\right\}
$$

such that $t_{i} \geqslant T$ and $d\left(x_{i} \cdot t_{i}, x_{i+1}\right)<\varepsilon$ for all $i$. Let $x_{0} * t$ denote the point on this chain $t$ units from $x_{0}$, i.e., if $t \geqslant 0$ then

$$
x_{0} * t=x_{i} \cdot\left(t-\sum_{n=0}^{i-1} t_{n}\right)
$$

where $\sum_{n=0}^{i-1} t_{n} \leqslant t<\sum_{n=0}^{i} t_{n}$ and if $t<0$ then

$$
x_{0} * t=x_{i} \cdot\left(t+\sum_{n=i}^{-1} t_{n}\right)
$$

where $-\sum_{n=i}^{-1} t_{n} \leqslant t<-\sum_{n=i-1}^{-1} t_{n}$. If $a, b \in R$, define

$$
x_{0} *[a, b]=\bigcup_{t \in[a, b]}\left\{x_{0} * t\right\}
$$

Given an infinite $(\varepsilon, T)$-chain $x_{0} * R$ define its $\omega$-limit set by

$$
\omega\left(x_{0} * R\right)=\bigcap_{t>0} \mathrm{Cl}\left(x_{0} *[t, \infty)\right) .
$$

Given $x, y \in M$ and $\varepsilon, T>0$, an $(\varepsilon, T)$-chain from $x$ to $y$ is a finite sequence of points and times, as above, with $x_{0}=x$ and $x_{n}=y$. Let $\mathscr{P}(f)$ $\equiv\{(x, y) \in M \times M \mid$ for any $\varepsilon, T>0$ there is an $(\varepsilon, T)$-chain from $x$ to $y\} ; \mathcal{P}$ is a closed subset of $M \times M$ and is a transitive relation. The chain recurrent set $\mathcal{R}(f)$ is $\{x \in M \mid(x, x) \in \mathcal{P}(f)\}$. $\Re$ is a closed invariant set containing $\Omega$. For 
$\Lambda \subset M$ we say $\left.f\right|_{\Lambda}$ is chain recurrent if $\Lambda$ is a compact invariant set and $\Re\left(\left.f\right|_{\Lambda}\right)=\Lambda$. $\mathscr{P}(f)$ induces an equivalence relation on $R(f)$. For $x, y$ $\in \mathcal{R}(f), x$ is equivalent to $y$ (written $x \sim y$ ) if $(x, y) \in \mathcal{P}(f)$ and $(y, x)$ $\in \mathscr{P}(f)$. Conley [2] shows

Proposition 2.1. The equivalence classes under $\sim$ are precisely the connected components of $\Re(f)$. And if $\Lambda$ is a component of $\mathcal{R}(f)$ then $\mathcal{P}\left(\left.f\right|_{\Lambda}\right)=\Lambda \times \Lambda$, i.e., the $(\varepsilon, T)$-chains between points of $\Lambda$ can be chosen to lie in $\Lambda$.

Consequently $\mathcal{R}\left(\left.f\right|_{\Re}\right)=\mathscr{R}(f)$, i.e., $\left.f\right|_{\Re}$ is chain recurrent. Also, the components of $\mathcal{R}$ are the maximal connected subsets of $M$ such that $f$ restricted is chain recurrent.

A closed invariant set $\Lambda \subset M$ is hyperbolic if the tangent flow $T f_{t}$ leaves invariant a continuous splitting $T_{\Lambda} M=E^{s} \oplus E^{u} \oplus E$ where, for some $\lambda$ $\in(0,1)$ and some Riemannian metric,

(i) if $v \in E^{u}$ and $t>0$ then $\left|T f_{t}(v)\right|>\lambda^{-t}|v|$,

(ii) if $v \in E^{s}$ and $t>0$ then $\left|T f_{t}(v)\right|<\lambda^{t}|v|$,

(iii) $E$ is the span of the vectorfield of $f$.

Stable manifold theory for a hyperbolic invariant set asserts, for each $x \in \Lambda$, the existence of $\alpha$-disks $W_{\alpha}^{s}(x)$ and $W_{\alpha}^{u}(x)$ which are tangent to $E_{x}^{s}$ and $E_{x}^{u}$. These families of disks are invariant; and there is a $\lambda \in(0,1)$ such that

$$
\begin{aligned}
& W_{\alpha}^{s}(x)=\left\{y \in M \mid d(x \cdot t, y \cdot t)<\alpha \lambda^{t} \text { for all } t>0\right\} \\
& W_{\alpha}^{u}(x)=\left\{y \in M \mid d(x \cdot t, y \cdot t)<\alpha \lambda^{-t} \text { for all } t<0\right\}
\end{aligned}
$$

Let

$$
W_{\alpha}^{s}(\Lambda)=\bigcup_{x \in \Lambda} W_{\alpha}^{s}(x) \text { and } W_{\alpha}^{u}(\Lambda)=\bigcup_{x \in \Lambda} W_{\alpha}^{u}(x) .
$$

$\Lambda$ is said to have local product structure if there is an $\alpha>0$ such that $W_{\alpha}^{u}(\Lambda) \cap W_{\alpha}^{s}(\Lambda)=\Lambda$.

With certain hyperbolicity assumptions it is possible to approximate infinite $(\varepsilon, T)$-chains with actual orbits. More precisely, an orbit $y \cdot R$ is said to $\delta$-trace an infinite $(\varepsilon, T)$-chain $x_{0} * R$ if there is an orientation preserving homeomorphism $g$ of $R$ fixing the origin such that $d\left(x_{0} * t, y \cdot g(t)\right)<\delta$ for all $t \in R$. We call $g$ a reparameterization of $y \cdot R$. In [3] we show

Proposition 2.2. Let $\Lambda$ be a hyperbolic closed invariant set. Given $\delta>0$ and $\alpha>0$ there is an $\varepsilon>0$ so that each $(\varepsilon, 1)$-chain in $\Lambda$ can be $\delta$-traced by some $x \in W_{\alpha}^{s}(\Lambda) \cap W_{\alpha}^{u}(\Lambda)$.

Proposition 2.3. If $\Lambda$ is a hyperbolic closed invariant set and $\left.f\right|_{\Lambda}$ is chain recurrent, then $\Lambda$ is contained in the closure of the set of periodic orbits of $f$.

Proposition 2.4. If $\Lambda$ is a hyperbolic closed invariant set then there exists $\delta>0$ so that:

(1) If $x, y \in \Lambda$ and $\left[t_{1}, t_{2}\right]$ is an interval containing zero and $g$ is a reparameterization of $y \cdot\left[t_{1}, t_{2}\right]$ with $d(x \cdot t, y \cdot g(t))<\delta$ for all $t \in\left[t_{1}, t_{2}\right]$, then

$$
|t-g(t)|<1 .
$$


(2) For each $\beta>0$ there is $S>0$ such that, if $x, y \in \Lambda$ and $g$ is a reparameterization of $y \cdot R$ with $d(x \cdot t, y \cdot g(t))<\delta$ for all $t$ belonging to an interval I where $I \cap g(I)$ contains $[-S, S]$, then $d(x, y \cdot r)<\beta$ for an $r$ with $|r|<1$. Moreover, if $I \cap g(I)=R$ then $x=y \cdot r$.

Part (2) of Proposition 2.4 establishes a type of flow expansiveness which says, roughly, that if two orbits are close enough for long enough time then segments of these orbits are much closer.

\section{Abstract $\omega$-limit sets are chain recurrent.}

THEOREM 3.1. Let $f$ be a flow on a compact metric space $\Lambda$. Then the following three conditions are equivalent:

(1) $f$ on $\Lambda$ is an abstract $\omega$-limit set.

(2) There is no proper open subset $U$ of $\Lambda$ with $U \neq \varnothing$ such that $(\mathrm{Cl} U) \cdot T$ $\subset U$ for some $T>0$.

(3) $\Lambda$ is connected and $\left.f\right|_{\Lambda}$ is chain recurrent.

We will show $(1) \Rightarrow(2) \Rightarrow(3) \Rightarrow(1)$. The arguments are flow versions of Bowen's Theorem 1 [1] plus the following lemmas:

LemMa 3.2. Let $\Lambda$ be connected and $\left.f\right|_{\Lambda}$ be chain recurrent. Then given $\varepsilon>0$ there is an infinite $(\varepsilon, 1)$-chain $x_{0} * R$ such that $\omega\left(x_{0} * R\right)=\alpha\left(x_{0} * R\right)=\Lambda$. In addition, given any $\varepsilon^{\prime}, T^{\prime}>0$ there is an $S>0$ such that $x_{0} *[S, \infty)$ and $x_{0}$ $*(-\infty,-S]$ are $\left(\varepsilon^{\prime}, T^{\prime}\right)$-chains.

Proof. Given $\varepsilon>0$, let $\left\{\varepsilon_{i}\right\}$ and $\left\{T_{i}\right\}$ be sequences with $\varepsilon_{i}<\varepsilon$ and $T_{i}>1$ such that $\varepsilon_{i} \rightarrow 0$ and $T_{i} \rightarrow \infty$ as $i \rightarrow \infty$. For each positive integer $i$ pick an $\varepsilon_{i}{ }^{-}$ dense set of points in $\Lambda$ and, by Proposition 2.1, construct a finite $\left(\varepsilon_{i}, T_{i}\right)$-chain connecting all these points. String all of these chains together to get an infinite $(\varepsilon, 1)$-chain with the desired properties.

LEMMA 3.3. Let $U$ be a nonempty, open, proper subset of $\Lambda$ compact with $(\mathrm{Cl} U) \cdot T \subset U$ for some $T>0$. Then $U^{\prime}=\cup_{t \geqslant 0} U \cdot t$ is a positively invariant, open, proper subset of $\Lambda$ with $\left(\mathrm{Cl} U^{\prime}\right) \cdot T \subset U^{\prime}$.

Proof. Clearly $U^{\prime}$ is open and positively invariant. $U^{\prime}=\cup_{0 \leqslant t \leqslant T} U \cdot t$ since $(\mathrm{Cl} U) \cdot T \subset U$; and $\mathrm{Cl} U^{\prime}=\cup_{0 \leqslant t \leqslant T}(\mathrm{Cl} U) \cdot t$ since $[0, T]$ is compact. Thus

$$
\begin{aligned}
\left(\mathrm{Cl} U^{\prime}\right) \cdot T & =(\underset{0 \leqslant t \leqslant T}{\bigcup}(\mathrm{Cl} U) \cdot t) \cdot T \\
& =\bigcup_{0 \leqslant t \leqslant T}((\mathrm{Cl} U) \cdot T) \cdot t \subset \bigcup_{0 \leqslant t \leqslant T} U \cdot t=U^{\prime}
\end{aligned}
$$

To show $U^{\prime}$ is proper assume $U^{\prime}=\Lambda$. Let $x_{0} \in \Lambda-\mathrm{Cl} U \neq \varnothing$ and $x_{i}$ $=x_{0} \cdot(i T)$ for $i=-1,-2, \ldots$ Each $x_{i} \in \Lambda-\mathrm{Cl} U$ since $(\mathrm{Cl} U) \cdot T \subset U$. Since $x_{i} \in U^{\prime}=\cup_{0 \leqslant t \leqslant T} U \cdot t$ there is a $y_{i} \in U$ such that $y_{i} \cdot t=x_{i}$ for some $0 \leqslant t \leqslant T$. Note that $y_{i} \cdot T \in U \cdot T$. Let $\alpha>0$ be a lower bound for the time it takes points to flow from $(\mathrm{Cl} U) \cdot T$ to $\Lambda-U$. The amount of time the orbit from $x_{-1}$ to $x_{0}$ spends in $U \cdot T$ is less than $T-2 \alpha$. Since the amount of time the orbit from $x_{i}$ to $x_{i+1}$ spends in $U$ is less than or equal to the amount the orbit from $x_{i+1}$ to $x_{i+2}$ spends in $U \cdot T$, the amount of time the orbit from 
$x_{i}$ to $x_{i+1}$ spends in $U \cdot T$ decreases by at least $2 \alpha$. Iterating this procedure shows that eventually there are no points of $U \cdot T$ between $x_{i}$ and $x_{i+1}$, which is a contradiction. Thus $U^{\prime}$ is proper.

Proof of Theorem 3.1. (1) $\Rightarrow$ (2). Let $g$ be a flow on $X$ and $h$ be the conjugacy between $\left.g\right|_{\omega(x)}$ and $\left.f\right|_{\Lambda}$. Suppose $U$ is a nonempty, open, proper subset of $\Lambda$ with $(\mathrm{Cl} U) \cdot T \subset U$. By Lemma 3.3, $U^{\prime}=U_{t \geqslant 0} U \cdot t$ has the same properties as $U$ plus being positively invariant. Let $V=h\left(U^{\prime}\right) . V$ is a nonempty, open, proper subset of $\omega(x)$ which is positively invariant. Since $\mathrm{Cl}(V)$ is compact and $\left(\mathrm{Cl} U^{\prime}\right) \cdot T \subset U^{\prime}$, there is a $P>0$ such that $(\mathrm{Cl} V) \cdot P$ $\subset V$. Again by Lemma 3.3, $V^{\prime}=\cup_{t \geqslant 0} V \cdot t$ is a positively invariant, open, proper subset of $\omega(x)$ with $\mathrm{Cl} V^{\prime} \cdot P \subset V^{\prime}$. Hence $\mathrm{Cl} V^{\prime} \neq \omega(x)$.

Let $y \in \omega(x)-\mathrm{Cl} V^{\prime}, z \in V^{\prime}, \alpha=d\left(y, \mathrm{Cl} V^{\prime}\right)>0$, and

$$
\beta=d\left(\omega(x)-\mathrm{Cl} V^{\prime}, \mathrm{Cl}\left(V^{\prime} \cdot P\right)\right)>0 .
$$

Let $\gamma>0$ be such that if $d(p, q)<\gamma$ then $d(p \cdot t, q \cdot t)<\frac{1}{2} \min \{\alpha, \beta\}$ for all $t$ with $0 \leqslant t \leqslant P$. Choose $S>0$ such that $d(x \cdot[S, \infty), \omega(x))<\gamma$. Since $z \in \omega(x)$ there is a time $S^{\prime}>S$ such that $d\left(x \cdot S^{\prime}, z\right)<\gamma$. Now $d\left(x \cdot\left(t+S^{\prime}\right), z \cdot t\right)<\alpha / 2$ implies $d\left(x \cdot\left(t+S^{\prime}\right), y\right)>\alpha / 2$ for $0 \leqslant t \leqslant P$. $d\left(x \cdot\left(P+S^{\prime}\right), z \cdot P\right)<\beta / 2$ and $z \cdot P \in V^{\prime} \cdot P$,

$$
d\left(x \cdot\left(P+S^{\prime}\right), \omega(x)-V^{\prime}\right)>\beta / 2>\gamma
$$

Thus there is a point $z_{1}$ in $V^{\prime}$ such that $d\left(x \cdot\left(P+S^{\prime}\right), z_{1}\right)<\gamma$. Successively repeating the preceding argument for time intervals of length $P$ shows that $d(x \cdot t, y)>\alpha / 2$ for all $t>S^{\prime}$ which shows $y \notin \omega(x)$. This contradiction finishes $(1) \Rightarrow(2)$.

Proof of (2) $\Rightarrow(3)$. If $\Lambda$ were not connected then the open-closed sets of a separation contradict (2). To show $\Lambda$ is chain recurrent take $\varepsilon, T>0$ and $x_{0}$ $\in \Lambda$. We will construct an $(\varepsilon, T)$-chain from $x_{0}$ to itself. Take a finite open $\varepsilon / 2$-cover of $\Lambda$. Let $U_{0}$ and $U_{1}$ be sets in this cover which contain $x_{0}$ and $x_{0} \cdot T$, respectively. If $U_{1}=U_{0}$ we are done. Since $\mathrm{Cl} U_{1} \cdot T \nsubseteq U_{1}, U_{1} \cdot T$ meets another set $U_{2}$ in the cover. $\mathrm{Cl}\left(U_{1} \cup U_{2}\right) \cdot T \nsubseteq U_{1} \cup U_{2}$ so $\left(U_{1} \cup U_{2}\right) \cdot T$ meets another set $U_{3}$ in the cover. Continue this procedure until $U_{n}$ is equal to $U_{0}$. For each $i=0,1, \ldots, n-1$ there is a point $x_{i}$ in $U_{0} \cup \cdots \cup U_{i}$ such that $x_{i} \cdot T \in U_{i+1}$. So there is an $(\varepsilon, T)$-chain from $x_{i}$ to any point in $U_{i+1}$. By induction one can chain from $x_{0}$ to any point in $U_{i+1}$; and hence there is an $(\varepsilon, T)$-chain from $x_{0}$ to $x_{0}$.

Proof of $(3) \Rightarrow(1)$. Let $\left\{\cdots, x_{-1}, x_{0}, x_{1}, \ldots ; \ldots, t_{-1}, t_{0}, t_{1}, \ldots\right\}$ be an infinite $(\varepsilon, T)$-chain with $\omega\left(x_{0} * R\right)=\alpha\left(x_{0} * R\right)=\Lambda$ as guaranteed in Lemma 3.2. Embed $\Lambda$ in the Hilbert cube $C$ and form the Cartesian product $C \times[0,1]^{2}$. For each integer $i$, define

$$
P_{i}= \begin{cases}x_{i} \cdot\left[0, t_{i}\right] \times(1 /(i+1), 0) & \text { if } i \geqslant 0 \\ x_{i} \cdot\left[0, t_{i}\right] \times(1 / i, 0) & \text { if } i<0\end{cases}
$$




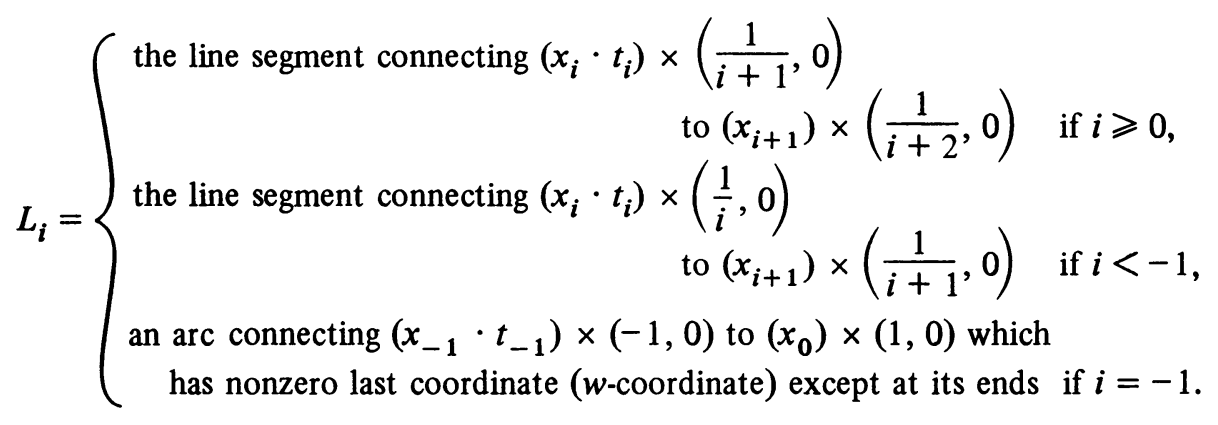

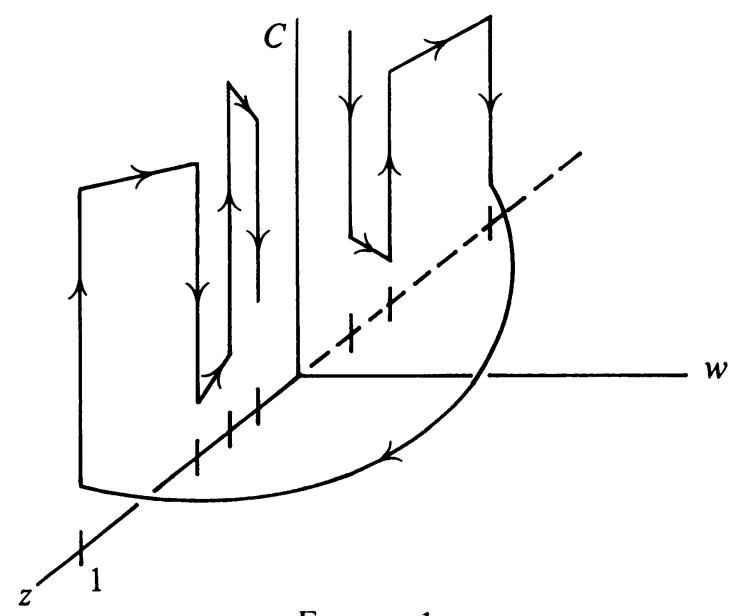

FIGURE 1

Let $Y=(\Lambda, 0,0) \cup \cup_{i=1}^{\infty}\left(P_{i} \cup L_{i}\right)$. We will define a flow on $Y$ (see Figure 1) such that one point will have its $\alpha$ - and $\omega$-limit sets equal to $(\Lambda, 0,0)$ which shows that $\Lambda$ is an abstract $\omega$-limit set. Define $g$ on $(\Lambda, 0,0)$ by $g_{t}(x, 0,0)$ $=\left(f_{t}(x), 0,0\right)$. On $P_{i}$ let $g$ be the flow induced by $f$. On $L_{i}$ let $g$ be the flow parameterized by arc length starting at $P_{i}$ and going to $P_{i+1}$. The only difficulty with the continuity of $g$ is for sequences of points, not in $\Lambda$, converging to $\Lambda$. But for a fixed $T>0$ a point close enough to $\Lambda$ will traverse at most one $L_{i}$ of small arc length. Hence the continuity of $g$ follows from that of $f$.

Finally, for any point $y \in Y-(\Lambda, 0,0), \omega(y)=\alpha(y)=(\Lambda, 0,0)$ since $\omega\left(x_{0} * R\right)=\alpha\left(x_{0} * R\right)=\Lambda$ and the arc lengths of the $L_{i}$ 's go to zero as $i \rightarrow \pm \infty$.

4. Chain recurrent and basic sets. The following theorem generalizes Bowen's result concerning abstract $\omega$-limit sets being actual $\omega$-limit sets for Axiom A diffeomorphisms.

THEOREM 4.1. Let $f$ be a smooth flow on $M$ and let $\Lambda$ be a hyperbolic closed invariant subset. If $\left.f\right|_{\Lambda}$ is an abstract $\omega$-limit set and $\alpha>0$, then there is an $x \in W_{\alpha}^{u}(\Lambda) \cap W_{\alpha}^{s}(\Lambda)$ such that $\alpha(x)=\omega(x)=\Lambda$.

Proof. Let $N$ be a closed neighborhood of $\Lambda$ whose maximal closed 
invariant subset $\Lambda^{\prime}$ is hyperbolic [4]. Take $\alpha>0$ and, without loss of generality, assume the $\alpha$ neighborhood of $\Lambda$ is contained in $N$. This insures that $W_{\alpha}^{u}(\Lambda) \cap W_{\alpha}^{s}(\Lambda) \subset \Lambda^{\prime}$.

Let $\delta$ be the number guaranteed in Proposition 2.4 and let $\varepsilon>0$ be the number in Proposition 2.2 corresponding to $\delta / 2$ and $\alpha$. By Theorem 3.1, $\Lambda$ is connected and $\left.f\right|_{\Lambda}$ is chain recurrent. Let $x_{0} * R$ be an infinite $(\varepsilon, 1)$-chain with $\omega\left(x_{0} * R\right)=\alpha\left(x_{0} * R\right)=\Lambda$ as constructed in Lemma 3.2. By Proposition 2.2 there is an $x \in W_{\alpha}^{u}(\Lambda) \cap W_{\alpha}^{s}(\Lambda) \subset \Lambda^{\prime}$ which $\delta / 2$-traces $x_{0} * R$. What we need to show is that $\omega(x)=\omega\left(x_{0} * R\right)$ and similarly that $\alpha(x)=\alpha\left(x_{0} * R\right)$.

Given $\varepsilon_{1}>0$ let $\beta>0$ be such that if $d(p, q)<\beta$ then $d(p \cdot t, q \cdot t)<\varepsilon_{1}$ for $|t| \leqslant 1$. Let $S>1$ be the number guaranteed in Proposition 2.4(2) corresponding to this $\beta$. By our choice of $x_{0} * R$ there is a time $T>0$ such that if $t>T$ then $x_{0} *[t-2 S, t+2 S]$ consists of at most two orbit segments and, since the jump is small, $d\left(\left(x_{0} * t\right) \cdot s, x_{0} *(t+s)\right)<\delta / 2$ for $s \in[-2 S$, $2 S]$. Thus since $x \cdot R \delta / 2$-traces $x_{0} * R, d\left(x \cdot g(t+s),\left(x_{0} * t\right) \cdot s\right)<\delta$ for $s$ $\in[-2 S, 2 S]$. Let $h(s)=g(t+s)-g(t)$; thus $d\left((x \cdot g(t)) \cdot h(s),\left(x_{0} * t\right) \cdot s\right)$ $<\delta$ for $s \in[-2 S, 2 S]$. Proposition 2.4(1) implies that $|h(s)-s|<1$ for $s$ $\in[-2 S, 2 S]$, hence $h([-2 S, 2 S])$ contains $[-S, S]$. Thus there is an $|r|<1$ such that $d\left(x_{0} * t,(x \cdot g(t)) \cdot r\right)<\beta$. Applying $f_{-r}$ gives

$$
d\left(\left(x_{0} * t\right) \cdot(-r), x \cdot g(t)\right)<\varepsilon_{1} .
$$

So after time $T$ every point on the orbit of $x$ is within $\varepsilon_{1}$ of some point on $x_{0} * R$ and vice versa. Therefore $\omega(x)=\omega\left(x_{0} * R\right)=\Lambda$ and similarly $\alpha(x)$ $=\alpha\left(x_{0} * R\right)=\Lambda$.

If we can show that the $x$ in this theorem is in $\Lambda$ then the orbit of $x$ is dense in $\Lambda$.

CoRollary 4.2. Let $f$ be a smooth flow on $M$, and let $\Lambda$ be a hyperbolic connected subset such that $\left.f\right|_{\Lambda}$ is chain recurrent. If $\Lambda$ has local product structure then it is topologically transitive.

Like Bowen [1], we will use the tracing theorem to obtain an isolating neighborhood for $\Omega$ in the Axiom A flow case.

THEOREM 4.3. If $\Lambda$ is a hyperbolic closed invariant set for $f$ with local product structure then it has a fundamental neighborhood, i.e., there is an open neighborhood $U$ of $\Lambda$ such that $\cap_{t \in R} U \cdot t=\Lambda$.

Proof. Since $\Lambda$ has local product structure there is an $\alpha>0$ such that $W_{\alpha}^{s}(\Lambda) \cap W_{\alpha}^{u}(\Lambda)=\Lambda$. Let $V$ be a neighborhood of $\Lambda$ whose maximal closed invariant subset $\Lambda^{\prime}$ is hyperbolic. Let $\delta$ be the number guaranteed in Proposition 2.4 with $\Lambda^{\prime}$ being the hyperbolic set. Proposition 2.2 gives an $\varepsilon>0$ depending on $\alpha$ and $\delta / 2$ so that every $(\varepsilon, 1)$-chain in $\Lambda$ can be $\delta / 2$-traced by a point in $W_{\alpha}^{s}(\Lambda) \cap W_{\alpha}^{u}(\Lambda)$. Choose $\beta, 0<\beta<\varepsilon / 2$, such that if $d(x, y)$ $<\beta$ then $d(x \cdot t, y \cdot t)<\min \{\delta / 2, \varepsilon / 2\}$ for all $|t| \leqslant 1$. Let $U$ be a $\beta$ neighborhood of $\Lambda$ contained in $V$. We will show that an orbit remaining in $U$ for all time is actually in $\Lambda$ by constructing an $(\varepsilon, 1)$-chain in $\Lambda$ which it $\delta / 2$-traces and then using Proposition 2.4(2).

Assume $y \cdot R \subset U$. Take $\left\{x_{i}\right\} \subset \Lambda$ with $d\left(x_{i}, y \cdot i\right)<\beta$ for each integer $i$. 
This gives an $(\varepsilon, 1)$-chain $x_{0} * R$ with $t_{i}=1$ since

$$
d\left(x_{i} \cdot 1, x_{i+1}\right) \leqslant d\left(x_{i} \cdot 1, y \cdot(i+1)\right)+d\left(y \cdot(i+1), x_{i+1}\right)<\varepsilon / 2+\beta<\varepsilon .
$$

The orbit of $y \delta / 2$-traces $x_{0} * R$. Since $x_{0} * R$ is an $(\varepsilon, 1)$-chain in $\Lambda$, there is an $x \in W_{\alpha}^{s}(\Lambda) \cap W_{\alpha}^{u}(\Lambda)=\Lambda$ which $\delta / 2$-traces $x_{0} * R$. Hence $x \cdot R \delta$-traces $y \cdot R$ and by Proposition 2.4(2) they are equal. Thus $y \cdot R \subset \Lambda$ and the proof is completed.

These results lead to the following theorem concerning basic sets.

THEOREM 4.4. $\Lambda$ is a basic set for a flow $f$ if and only if

(a') $\Lambda$ is compact and invariant,

(b') $\Lambda$ is hyperbolic,

(c') $\left.f\right|_{\Lambda}$ is chain recurrent,

(d') $\Lambda$ is connected,

( $\left.\mathrm{e}^{\prime}\right) \Lambda$ has local product structure.

Proof. Clearly Smale's definition of a basic set implies $\left(a^{\prime}\right)-\left(d^{\prime}\right)$, and $\left(e^{\prime}\right)$ follows from the existence of an isolating neighborhood.

To prove the opposite implication note that Corollary 4.2 gives (d) and Theorem 4.3 gives (e). Proposition 2.3 implies that $\Lambda$ is contained in the closure of the set of periodic orbits. The proof of Proposition 2.3 used Proposition 2.2 to construct tracing periodic orbits. Since $\Lambda$ has local product structure, these periodic orbits are contained in $\Lambda$. This gives (c) and finishes the theorem.

A hyperbolic component of $\mathcal{R}(f)$ satisfies $\left(a^{\prime}\right)-\left(d^{\prime}\right)$ and also has local product structure because of the following proposition. Consequently, a hyperbolic component of $\mathscr{R}(f)$ is a basic set.

Proposition 4.5. Let $\Lambda$ be a hyperbolic component of $\Re(f)$ then $W^{s}(\Lambda)$ $\cap W^{u}(\Lambda)=\Lambda$.

Proof. Let $x \in W^{s}(\Lambda) \cap W^{u}(\Lambda)$. For each $y \in \Lambda$ we will show $x \sim y$ so $x \in R(f)$ and, in particular, $x \in \Lambda$ by Proposition 2.1. Since $x \in W^{s}(z)$ for some $z \in \Lambda$, construct a chain from $x$ to $z \cdot t$ for some large $t$ and then from $z \cdot t$ to $y$ in $\Lambda$. Similarly, $x \in W^{u}\left(z^{\prime}\right)$ for some $z^{\prime} \in \Lambda$ so construct a chain from $y$ to $z^{\prime} \cdot(-t)$ for some large $t$ and then jump to the orbit of $x$.

\section{REFERENCES}

1. R. Bowen, w-limit sets for Axiom A diffeomorphisms, J. Differential Equations 18 (1975), 333-339.

2. C. Conley, The gradient structure of a flow: I, IBM Research, RC 3932 (\#17806), Yorktown Heights, New York, July 17, 1972.

3. J. Franke and J. Selgrade, Hyperbolicity and chain recurrence (submitted).

4. M. W. Hirsch, J. Palis, C. Pugh and M. Shub, Neighborhoods of hyperbolic sets, Invent. Math. 9 (1969/70), 121-134. MR 41 \# 7232.

5. C. Pugh and M. Shub, The $\Omega$-stability theorem for flows, Invent. Math. 11 (1970), 150-158. MR 44 \# 4782.

6. S. Smale, Differentiable dynamical systems, Bull. Amer. Math. Soc. 73 (1967), 747-817. MR 37 \#3598; erratum, 39, p. 1593.

Department of Mathematics, North Carolina State University, Raleigh, North CaroLINA 27607 